\title{
Las relaciones sociales como recurso estratégico para el desarrollo de la sociedad. El capital social disponible en Andalucía y en Cataluña
}

\author{
Mercedes Camarero Rioja \\ Universidad Pablo de Olavide. Sevilla (Spain) \\ mmcamrio@upo.es
}

Recibido: 24-07-2008

Aceptado: 07-10-2009

\section{Resumen}

En este trabajo se contabiliza el capital social disponible en Andalucía y en Cataluña, y se detalla cuánto contribuye, cada una de las dos formas de capital — vinculante y aglutinante- - a la producción de dicho stock. El interés último es dar cuenta de los recursos disponibles como medida de la calidad de la sociedad. El instrumento analítico del que nos servimos es un índice sintético, el índice sintético de capital social (ISCS), y dos índices específicos. Estos índices contribuyen a describir tres sistemas de producción de capital: el modelo europeo, el modelo de la Europa del Este y el modelo español al que pertenecen las comunidades autónomas de Cataluña y Andalucía. Este sistema se caracteriza por un elevado índice de capital social (ISCS España $=4,08$ ) equivalente al europeo (ISCS Europa = $3,96)$ y por el menor peso relativo del capital social cívico. Aunque el capital social disponible en Andalucía es prácticamente igual al ISCS de Cataluña, algunas de las diferencias encontradas nos conducen a describir dos variantes del modelo.

Palabras clave: capital social, índice sintético, calidad social, capital social vinculante, capital social aglutinante, sociología comparativa, Andalucía, Cataluña, España, Europa.

Abstract. Social relations as a strategic ressourse for the development of society. The available social capital in Andalusia and Catalonia

Social Capital is an informal rule which promotes cooperation between individuals. This reciprocity rule, based on trust, is useful for individuals as well as for society. In fact, social capital has entailed numerous social advantages; it reduces the typical costs of market economies — such as contract, surveillance or punishment - and offers the best guarantee for democracy. Consequently, we consider which kind of relationships can be a strategic resource for society development.

To calculate how much social capital is available in an European country or in an European region, I have created a composite index. This index, based on Putnam's Social Capital Index, consists of 17 social indicators relating to 4 dimensions. Our SCI (Social Capital Index) is an additive index, but is useful also to distinguish between «bridging» and «bonding» social capital. In short, we can calculate the "Social-Gross Domestic Product» and know the relative contribution to S-GDP for «bridging», based on civic relationships, and «bonding», based on friendship and familiar relationships, sub-indices. Therefore, I have estimated the SCI and its sub-indices for two Spanish regions -Andalusia and Catalonia-, for Spain, for the European Union (UE27), Western Europe (UE15) and Eastern Europe (UE27-UE15). 
According to data, in Europe there are three social capital production systems: the European system, the Eastern European system, and the Spanish system which includes the Andalusian and the Catalan types. Spain $(\mathrm{SCI}=4.08)$ produces as much social capital as Europe $(\mathrm{SCI}=3.96)$; however, it produces less civic social capital than Europe. Contrary to Spain, the newest countries of the European Union obtain the lowest index. The Spanish model combines the highest production of social capital with the lowest rate of civic capital. In Spain, and also in Andalusia and Catalonia, primary relationships, such as family relations, produce much more social capital than secondary relationships, such as civic relations. The Andalusian system has more exaggerated «Spanish traits» than the Catalonian one.

Key words: Social Capital Index, Composite Index, social quality, bridging social capital, bonding social capital, comparative sociology, Andalusia, Catalonia, Spain, Europe.

\section{Sumario}

1. El capital social en la sociedad contemporánea

2. Las relaciones sociales como recurso colectivo

3. Las relaciones sociales como recurso estratégico para el desarrollo de la sociedad

4. El índice sintético de capital social (ISCS)
5. El capital social disponible en Andalucía y en Cataluña

6. Los modelos poductivos de capital social

7. Los modelos productivos de capital social en Andalucía y Cataluña

Referencias bibliográficas

\section{El capital social en la sociedad contemporánea}

El reciente interés académico por el estudio de las normas que promueven la cooperación entre los individuos y producen bienes públicos coincide tanto con el interés político por fomentarlo, como con la alerta a propósito de su declive. El individualismo es uno de los factores de cambio comunes, tanto al proceso de modernización como al de postmodernización de las sociedades. El título de la conocida obra de Putnam (2002), Solo en la bolera, alude a este fenómeno, mientras que el subtítulo, Declive y resurgimiento de la comunidad norteamericana, hace luz sobre la transformación de la naturaleza de las relaciones sociales fuente del capital social.

El interés desde la sociología se traduce en un incremento exponencial del número de artículos publicados sobre el tema. Así, por ejemplo, la base Sociological abstracts contiene un total de 1.442 artículos, en cuyo título figura el término "capital social», de los que tan sólo 38 están publicados antes de 1990. Y mientras que, entre 1990 y 1994, se publican 5 artículos por año, en cada uno de los dos quinquenios siguientes vieron la luz un número medio anual de 40 y 143 artículos, respectivamente, para alcanzar, en el trienio 20052007, los 150 artículos al año.

Entre las medidas políticas que expresan la definitiva toma de conciencia a propósito de la utilidad de promover, crear o construir capital social, destaca- 
mos, por su envergadura, el proyecto de la Comisión de la Unión Europea para la elaboración del sistema europeo de indicadores sociales de cohesión social. Se trata de un instrumento de medida que habrá de servir para monitorizar el avance de Europa en términos de cohesión social, lo que se traduce en medir simultáneamente cuánto se reduce la desigualdad social y se fomenta el capital social a base de fortalecer los vínculos sociales interpersonales (Berger-Schmitt, 2002).

La ciencia social contemporánea reacciona ante la moderna identificación entre progreso económico y bienestar social, para proclamar que la calidad de una sociedad no depende exclusivamente de sus logros económicos, sino también del grado en que ésta promueva la participación y la justicia sociales. Así, la cooperación social se instituye como recurso económico y político: hace más eficiente la vida social en la medida que minimiza los costes de transacción propios de las economías de mercado, al tiempo que se constituye en garantía de la existencia de una sociedad auténticamente democrática. Y, en ambos sentidos, el capital social de que dispone una sociedad es un recurso estratégico para su desarrollo y una medida de la calidad de la sociedad.

Este fenómeno contemporáneo debe ser analizado en el contexto de una sociedad europea que, a principios del siglo XXI, aunque moderna, muestra síntomas evidentes de encontrarse involucrada en un proceso de postmodernización. Si nos circunscribimos al ámbito concreto de las relaciones interpersonales, el contexto social desde el que estudiamos el capital social es el de una sociedad individualista que se halla inmersa en un proceso de individuación.

El proceso de modernización viene definido tanto por el incremento en el número de organizaciones de carácter burocrático, como por la creciente centralidad que éstas alcanzan en los ámbitos económico y político. El proceso hacia el individualismo ha sido propiciado por el declive de la comunidad, promotora de las identidades colectivas, y el auge de la sociedad, propiciadora de identidades individuales. Los individuos, a imagen y semejanza de las organizaciones formales en las que participan, establecen relaciones sociales secundarias, racionalmente diseñadas para alcanzar sus objetivos. De este modo, al analizar las relaciones sociales que producen capital del tipo social, se observa que es la razón instrumental la que mueve a las personas a participar en la producción de bienes públicos: la expectativa de que obtendrán como contrapartida un beneficio individual derivado del mayor bienestar colectivo (Herreros Vázquez, 2002).

En la postmodernidad, el proceso de individuación aparece vinculado, por una parte, con el progresivo descrédito de la autoridad de tipo burocrático, que promueve la menor aceptabilidad de la norma burocrática y, por otra, con el creciente énfasis en la libertad y la autonomía de los individuos (Inglehart, 2006). Se trata de un proceso por el que los sujetos se sienten más libres, más capaces, más dispuestos y con más derecho a decidir por ellos mismos en cualquier ámbito de su existencia. Dicho de otra forma, se hacen individuos y/o ciudadanos. Este cambio tiene consecuencias en el ámbito político, en el que uno de los mejores índices de calidad democrática es, precisamente, la participación plena de los ciudadanos en la vida política. 
En síntesis, la medida del capital social disponible se inserta en el marco de una sociedad individualizada y sometida a la presión de las demandas de mayor autonomía por parte de los individuos. En los términos del profesor Inglehart, el desarrollo económico genera no una, sino dos dimensiones importantes de variación transcultural, una vinculada a la industrialización, que se mueve en la dirección de la racionalización, la centralización y la burocratización, y otra vinculada al nacimiento de la sociedad postindustrial, que tiende a enfatizar la autonomía individual y los valores de la autoexpresión (Inglehart, 2006: 66).

\section{Las relaciones sociales como recurso colectivo}

La norma informal que promueve la cooperación entre los individuos (Fukuyama, 2001: 7) es producto de la sociabilidad humana, al mismo tiempo que productora de recursos que utilizan los individuos, los grupos y/o la sociedad en su conjunto. Este fenómeno social y económico se ha analizado desde tres perspectivas teóricas: desde el paradigma individualista, el culturalista y el relacional (Donati y Prandini, 2007; Requena, 2004). Si Coleman lo considera un recurso individual y se interesa por su contribución a la creación del capital humano (Coleman, 1988; Burt, 1997), Putnam y también Fukuyama lo entienden como una cualidad de los grupos y de la sociedad (Putnam, 2002; Fukuyama, 2001), lo cual promueve un análisis transcultural de las sociedades. Más recientemente, Donati y Prandini adoptan una actitud esencialista al definir el capital social como una propiedad y una cualidad de las relaciones sociales (Donati y Prandini, 2007).

Esta norma social se hace efectiva cuando los individuos deciden contribuir a la producción de determinados bienes públicos, a la espera de conseguir aquellos bienes individuales que se derivan del mayor bienestar colectivo. En consecuencia, la conocida técnicamente como norma de «reciprocidad generalizada» no debe confundirse ni con la «reciprocidad específica», que regula acuerdos concretos entre particulares, ni con el altruismo, puesto que el don y la reciprocidad son términos esencialmente incompatibles. Para que esta norma social sea capaz de regular de manera eficiente los intercambios diferidos en el tiempo, será condición sine qua non que los miembros de la sociedad confíen los unos en los otros, que se encuentren seguros de que todos cumplirán los compromisos.

Hanifan es el primer autor que introduce el concepto de capital social en un artículo publicado en 1916 y titulado «The Rural School Community Center». Consciente de su innovación y de la reacción que pueda provocar, apostilla, en la primera página, que la idea de capital social debe entenderse en sentido figurado, aunque concluye sin ambages que este término tiene todas las propiedades del capital económico: satisface necesidades de los individuos, es acumulativo y productivo (Hanifan, 1916: 130-138).

En su calidad de recurso productivo, tiene valor de uso y valor de cambio. Tiene valor de cambio porque, al reducir los costes de transacción derivados 
del funcionamiento de toda economía de mercado, contribuye a mejorar la eficiencia económica del sistema. Y, en la medida en que mejora la calidad democrática de una sociedad, que sólo puede alcanzarse a base de que los ciudadanos participen en el gobierno de la res publica, tiene valor de uso.

El capital social tiene carácter estructural porque su forma depende de la estructura de relaciones sociales en las que se produce (Coleman, 1988). La forma que adopte varía según la naturaleza de los vínculos que se establecen entre los individuos, del tipo de redes sociales y de las normas de reciprocidad y confianza derivadas de ellas (Putnam, 2002; Coleman, 1988). Las dos formas típico-ideales a las que se refiere Putnam son: el capital social vinculante (bridging - 'que tiende puentes'-) y el capital social aglutinante (bonding - 'que adhiere-). El capital social vinculante promueve la norma de la reciprocidad generalizada que se hace efectiva en la cooperación, por lo que constituye el verdadero capital social cívico. Por su parte, el capital social aglutinante promueve la reciprocidad específica y moviliza la solidaridad.

El capital social vinculante y el aglutinante se diferencian en tres aspectos. Según el «radio del círculo de confianza» en el que las normas se aplican, el capital social resultante puede ser incluyente o excluyente. Según que los individuos se adhieran de forma autónoma o heterónoma a la norma, el capital social les vincula o les obliga. Se distinguen, en tercer lugar, según cual sea la forma de apropiación y disfrute de los bienes, como bienes de consumo público o privado.

Fukuyama introduce el concepto radius of trust para poner en evidencia la correlación que existe entre el radio del círculo social en el que las normas cooperativas se aplican y el beneficio aportado a la sociedad. El radio del círculo de confianza puede circunscribirse a un grupo de interés o a la sociedad en su conjunto (Fukuyama, 2001: 8). En términos generales, dentro de las redes de sociabilidad informal, como los círculos familiares o de amistad, el CS tiende a ser excluyente - los bienes generados solo pueden ser consumidos o disfrutados por los miembros de la red. Los bienes públicos que tienen su origen en organizaciones de carácter secundario, como partidos políticos u organizaciones sindicales, tienden a ser incluyentes, en la medida que, una vez producido el bien, y con independencia de que se haya o no colaborado en su producción, nadie puede ser excluido del disfrute de su beneficio.

Putnam utiliza los sintagmas capital social «vuelto hacia dentro» y capital social «vuelto hacia fuera» para referirse a las distintas formas de apropiación del capital, como bien privado o público, respectivamente. Inglehart se refiere a la forma en que las normas se imponen a los individuos. Mientras que, en los círculos reducidos e íntimos, la confianza interpersonal une y obliga, la confianza generalizada que es más probable que surja en el seno de organizaciones formales y voluntarias, vincula (Inglehart, 2006).

Las relaciones sociales formales (Putnam, 2002), los vínculos secundarios (Coleman, 1988) o las asociaciones civiles (Pérez Díaz, 2003) tienden a producir capital cívico que se basa en una norma de reciprocidad generalizada, produce bienes de interés general y es inclusivo. Pero la cooperación puede dar 
lugar a consecuencias no deseadas que pueden ser incluso perversas, antisociales (Putnam, 1993, 2002) o incívicas (Pérez Díaz, 2003), y estas externalidades suelen estar asociadas con determinadas redes de sociabilidad informal.

\section{Las relaciones sociales como recurso estratégico para el desarrollo de la sociedad}

Las relaciones sociales constituyen un recurso estratégico, porque contribuyen a conseguir una mayor eficiencia de la vida económica y política de la sociedad. Por una parte, son útiles en tanto que facilitan la reducción de los costes de transacción propios de toda economía de mercado y, por otra, porque, al promover la vida asociativa necesaria para el éxito del gobierno en las democracias modernas, constituyen una garantía para la estabilidad y la calidad de las democracias liberales (Putnam, 2000; Fukuyama, 2001; Witheley, 2000; Paxton, 2002).

Esta tesis se enmarca en los más recientes desarrollos de la teoría sociológica. Así, Coleman, en Foundations of social theory, introduce el concepto de estructura social en su teoría de la acción racional (Coleman, 1994). Y, más concretamente, cuando señala que concebir el capital social como recurso para la acción es una vía para incluir la estructura social dentro de su paradigma de la acción racional (Coleman, 1988). Así Granovetter cuando, en los pioneros trabajos que señalan un antes y un después en el desarrollo de la sociología económica, afirma que la acción económica se encuentra incrustada (embedded) en una estructura de relaciones sociales desde la que debe ser explicada (Granovetter, 1985).

Whiteley, en el artículo "Crecimiento económico y capital social», pone en evidencia la importancia de las relaciones sociales, al sentenciar que el impacto que tiene el capital social sobre el crecimiento económico es al menos similar al del capital humano. Este autor detalla los mecanismos concretos por los que el capital social contribuye a promover un funcionamiento más eficiente de la economía (Whiteley, 2000). En la medida en que la confianza social facilita cierta desburocratización de los intercambios económicos, disminuye el coste de su planeamiento y ejecución, con lo cual se reducen, de esta forma, los costes de transacción. Si existen valores cívicos compartidos que promueven el cumplimiento de los acuerdos políticos coactivos, como los contenidos en un convenio colectivo, por ejemplo, se minimizan los costes derivados de tener que recurrir a la coacción para que se cumplan las normas. El capital social es bueno también para reducir las deseconomías del fraude y del robo. Esto es, minimiza los costes imputables a la prevención, la vigilancia y el castigo de tales comportamientos.

Fukuyama afirma que el capital social es la condición sine qua non para la estabilidad de la democracia liberal y Putnam aporta la evidencia empírica de la relación que existe entre sociedad civil y éxito en el desarrollo institucional (Putnam, 1993). En su argumentación, Fukuyama parte del riesgo que supone para la democracia liberal el excesivo individualismo. La exclusiva preocu- 
pación por la vida privada y la familia suele tener como consecuencia una escasa disposición a comprometerse en los asuntos públicos. Es necesario un stock abundante de capital social que promueva la verdadera sociedad civil, sin la que no podrá existir una sociedad auténticamente democrática. La sociedad civil resulta, así, un escalón intermedio entre individuos que, de no organizarse, bien se verían abocados a un estado de anarquía, bien se encontrarían sometidos a un estado omnímodo y autoritario que no garantizaría la libertad individual. La sociedad civil sirve de contrapeso para alcanzar el óptimo equilibrio entre el poder del estado y la protección de los individuos frente al poder del propio estado (Fukuyama, 2001: 11).

Putnam y sus colaboradores publican, en el año 1993, Making democracy work, un magnífico trabajo empírico en el que, durante años, investigan la relación entre el capital social comunitario y el funcionamiento de sus instituciones en diferentes regiones de Italia. La primera lección es que el contexto social y la tradición cívico-participativa constituyen profundos condicionamientos para la efectividad de las instituciones. La segunda, vinculada con la observación de los efectos de la implantación de los gobiernos regionales desde los años setenta, nos enseña que el cambio de las instituciones formales puede inducir una profunda transformación de las prácticas políticas. Si esto es cierto, se puede confiar en que, si se crea capital social, la democracia funcione mejor (Putnam, 1993: 185). En las regiones del norte de Italia, que se caracterizan por un mayor nivel de desarrollo económico y un mejor funcionamiento de las instituciones, las redes de compromiso cívico están estructuradas por vínculos horizontales. Y, al contrario, en las regiones del sur, los vínculos sociales se han estructurado verticalmente (Putnam, 1993: 181).

Los vínculos cívicos tienen una correlación directa y positiva con el desarrollo económico y con la calidad democrática. Los vínculos informales cumplen una función secundaria en términos de cambio y desarrollo de la sociedad, y contribuyen a la estabilidad social. Putnam se refiere a ambas funciones con esta ilustrativa cita de Xavier de Souza «el capital social bonding (aglutinante) es bueno para salir del paso, pero el capital social que tiende puentes es crucial para salir adelante» (Putnam, 2002: 23).

En la revisión que Ronald Inglehart hace de su conocida tesis sobre el cambio de valores, dedica una atención especial a las relaciones que se estipulan entre las dos formas del capital social y el nivel de desarrollo socioeconómico de la sociedad. En condiciones de necesidad, los valores materialistas de supervivencia acentúan la importancia del capital social aglutinante, mientras que, en condiciones de seguridad, tienden a propagarse el conjunto de actividades cooperativas y críticas con el poder que suelen relacionarse con el capital social vinculante. El cambio social en las sociedades occidentales avanzadas no estaría propiciando el declive del capital social, sino su transformación. Según Inglehart, el capital social estaría cambiando de forma, puesto que se reduciría el tipo específico de capital social aglutinante y aumentaría, a través de la propagación de actividades colectivas autoorganizadas y desafiantes para la élite, el capital social vinculante (Inglehart, 2006: 190-191). 
El énfasis en la elección humana autónoma es el nexo común entre los valores de la autoexpresión y la participación cívica. Si los valores de la supervivencia que promueven la desconfianza social, contribuyen al repliegue de la sociedad en torno a los círculos de sociabilidad primaria, los valores de la autoexpresión, que fomentan las actitudes procívicas, provocan la expansión del capital social civil.

Este es el marco analítico desde el que describimos las posibles diferencias que existen entre Andalucía y Cataluña en términos del capital social disponible. El grado en que, en una sociedad, se encuentran implantados los valores de la autoexpresión que fomentan las actitudes procívicas está vinculado con un alto nivel de seguridad económica. Desde la perspectiva que define las relaciones sociales como recursos para el desarrollo de la sociedad, podemos decir que los valores de conformidad asociados con el capital social aglutinante promocionan la estabilidad social, mientras que las actitudes procívicas ligadas con el capital social vinculante, promueven el cambio y la innovación sociales.

\section{El índice sintético de capital social (ISCS)}

El interés científico que despierta el capital social, hace ahora dos décadas, se acompaña con el desarrollo de algunos instrumentos de medida, entre los que destacan los índices sintéticos. En 1998, Onyx y Bullen, en un estudio pionero, elaboran el Social Capital Index. Se trata de un índice empírico sencillo basado en una lista de 36 preguntas sobre capital social (Onyx y Bullen, 1998). Robert Putnam publica, en el año 2000, su ya clásico trabajo sobre la evolución del capital social en los estados norteamericanos. Este trabajo, basado en el Social Capital Index, marca un importante hito científico a partir del que se inicia un vivo debate académico. Su trabajo se prolonga en el marco del denominado Saguaro Seminar: Civic Engagement in America, institución en la que se ha diseñado, por ejemplo, el Social Capital Impact Assessment, índice específico que se utiliza para conocer el impacto de la implementación de los programas de fomento del capital social. El Nicholas Petris Center (Petris Center on Health Care Markets and Consumer Welfare) elabora el Petris Social Capital Index (PSCI) como una medida sintética del CSC (Community Social Capital), a través del cual investiga los efectos del capital social sobre la salud cardiovascular o los hábitos de vida saludables (Scheffler, Brown y Rice, 2007). Dentro de nuestras fronteras, el colectivo IOÉ ha elaborado para España un índice sintético de participación ciudadana.

En el contexto de esta producción científica, y tomando en consideración todos los intentos precedentes para establecer un índice sintético, nuestro trabajo ha consistido en elaborar un índice sintético de capital social adaptado a la realidad social europea, a partir del Social Capital Index (SCI) de Putnam. El índice que presentamos aquí, el índice sintético de capital social (ISCS), se diferencia del concebido por el autor de Bowling alone en dos aspectos. Primero, se ha eliminado la dimensión "voluntariado de la comunidad», al considerar 
que mide un fenómeno singular de la cultura estadounidense. Segundo, la dimensión «participación en asuntos públicos» se ha desplegado en dos subdimensiones: "confianza institucional» y «participación política». El sistema se compone de un total de diecinueve indicadores, dos de los cuales son complementarios.

En consonancia con la definición estructural del concepto de capital social, el sistema de indicadores está estructurado en cuatro dimensiones vinculadas con las condiciones en las que este recurso se produce: la confianza social, la participación en la vida social comunitaria, la participación en la vida política y la sociabilidad informal. Se incluye una quinta dimensión, que no forma parte del sistema y que refleja el grado en que las actitudes procívicas se encuentran extendidas entre la población. Para medir el capital social disponible, se ha elaborado un índice sintético de capital social y dos índices específicos: el índice de capital social vinculante y el índice de capital social aglutinante. Estos índices están basados en un sistema de indicadores de capital social elaborado para monitorizar el cambio social en Andalucía (Bericat y Camarero, 2008). Y, puesto que de lo que se trata es de medir el stock de capital social disponible -concepto de naturaleza económica y acumulativa-, el índice es de naturaleza aditiva.

En primer término, se ha incluido la confianza social. Que los individuos confíen los unos en los otros es la condición sine qua non para que colaboren en la producción del bien común. La confianza social es también el resultado de dicha cooperación. Según nos enseña el dilema del prisionero, sólo si colaboran, los jugadores obtendrán el mejor resultado para ambos. Pero, puesto que los jugadores pueden estar motivados para obtener un mejor resultado individual si traicionan al otro, el mejor resultado colectivo sólo puede alcanzarse si, recíprocamente, uno confía en el otro. Esta dimensión se compone de tres indicadores de confianza horizontal o confianza interpersonal. Se han seleccionado tres de los cinco indicadores de la escala construida por Rosemberg. Se basan en preguntas de encuesta en las que se pide al entrevistado la opinión acerca de cómo espera que se comporte la gente. Lo que miden son las expectativas que las personas tienen sobre qué haría la "gente», el otro generalizado, en determinadas circunstancias.

La segunda dimensión, la de participación en la vida social comunitaria, mide la colaboración efectiva de los individuos en asociaciones secundarias y está formada por nueve indicadores. Mide el grado en que está extendida entre la población la disposición a cooperar con otros para alcanzar metas colectivas. Estos indicadores dan cuenta también de la intensidad de la cooperación, puesto que se ofrecen datos de pertenencia y de participación activa para cada una de las organizaciones consideradas.

La participación en la vida política es una dimensión sustantiva y central del capital social. La participación política revela la existencia de una auténtica sociedad civil, lo que cumple una función de garantía en una sociedad democrática. La implicación de los ciudadanos en la definición y decisión de las medidas que conducen al bienestar público da cuenta de la calidad democrá- 
tica de una sociedad. La participación en la vida política se ha traducido en dos subdimensiones: confianza institucional y participación política. La confianza en las instituciones cumple la misma función para la vida política que la confianza social cumple para la vida comunitaria, aunque una y otra confianzas obedecen a dos lógicas diferentes y, por tanto, los efectos que tengan sobre el capital social serán distintos. La participación política delegada a las instituciones representativas del estado y la participación política directa, como opinión pública, son los dos aspectos centrales de toda democracia sobre los que aportan conocimiento estos indicadores.

La cuarta dimensión, la sociabilidad informal, mide aquel capital social que es producto de las relaciones primarias que tienen lugar en el seno de grupos informales, como la familia, los amigos o los compañeros de trabajo. Y, unque el capital social así producido puede tener consecuencias cívicas o incívicas, según muestra Putnam, la participación en cualquier tipo de red social, incluidas las primarias, contribuye a fomentar la participación cívica. En esta dimensión, se incluyen cinco indicadores que dan cuenta de la participación en redes informales con familiares y compañeros de trabajo y de la intensidad del vínculo.

El índice sintético de capital social se calcula como la media de los valores de los indicadores en cada dimensión. El ISCS puede descomponerse, a su vez, en dos subíndices: el subíndice de capital social vinculante (bridging) mide el capital social cívico en el sentido estricto de su definición; el subíndice de capital social aglutinante (bonding) contabiliza, por su parte, lo que producen las redes primarias, así como otro conjunto de relaciones de carácter menos racional y más desinteresado. En síntesis, el stock de capital social disponible es la suma promedio de los subíndices de cada uno de los cuatro componentes del capital social.

\section{El capital social disponible en Andalucía y en Cataluña}

El cuadro 1 representa la estructura del sistema de indicadores de capital social ${ }^{1}$ y ofrece los valores de los 19 indicadores, de los subíndices y del índice de capital social para Andalucía y para Cataluña. Al objeto de disponer de un marco de referencia desde el que interpretar las diferencias existentes entre ambas comunidades autónomas, también se han consignado los datos de España y de Europa.

En el cuadro 2, figura la operacionalización de los subíndices de cada dimensión, la del subíndice de capital social vinculante y del subíndice de capital social aglutinante, así como la del índice sintético. Desde una concepción acumulativa del capital social, tanto los subíndices de los componentes del capi-

1. Este trabajo lo he desarrollado como miembro del equipo del proyecto de investigación Puesta en marcha del nuevo sistema de indicadores sociales de Andalucía y elaboración de un análisis de la situación social de la población andaluza, subvencionado por el Instituto de Estadística de Andalucía y cuyo investigador principal es D. Eduardo Bericat Alastuey. 
Cuadro 1. Estructura del índice sintético de capital social

\begin{tabular}{|c|c|c|c|c|c|}
\hline Indicadores & Europa & España & And. & Cat. & Fuentes \\
\hline D.1. Confianza social & 4,07 & 3,81 & 3,57 & 3,76 & \\
\hline $\begin{array}{l}\text { I1. «Se puede confiar en la mayoría } \\
\text { de la gente» (\% acuerdo: suma } \\
\text { categorías } 6-10) \text { (p: A8) }\end{array}$ & 37,4 & 41,8 & 37,7 & 40,7 & ESS-2007 \\
\hline $\begin{array}{l}\text { I2. «La mayoría de la gente sería } \\
\text { honrada conmigo" (\% acuerdo: } \\
\text { suma categorías } 6-10) \text { (p: A9) }\end{array}$ & 50,3 & 44,8 & 42,9 & 42,2 & ESS-2007 \\
\hline
\end{tabular}

I3. «La mayoría de las veces la gente intenta ayudar a los demás» (\% acuerdo: suma categorías 6-10) (p: A10)

$34,5 \quad 27,7 \quad 26,6 \quad 30,1 \quad$ ESS-2007

\section{D.2. Participación en la vida social} comunitaria

$2,20 \quad 2,11 \quad 1,81 \quad 2,05$

I4. Proporción de población activa que es miembro activo de, al menos, una organización (v206-v214)

$22,8 \quad 23,6 \quad 17,8 \quad 21,4 \quad$ ECVT-2004

I5. Proporción de población activa que es miembro activo en organizaciones en beneficio de la comunidad (v206-v214)

I6. Población mayor de 15 años que articipa en algún tipo de organización para mejorar el país (B15)

I7. Donaciones de sangre por 1.000 habitantes (\%o)

$\begin{array}{rrrrr}13,8 & 14,3 & 12,3 & 9,5 & \text { ECVT-2004 } \\ 42,4 & 37,1 & 33,3 & 38,7 & \text { FEDS-2006 } \\ & & & & \text { FIODS-01 }\end{array}$

\section{D.3. Participación en la vida política}

$\begin{array}{rrrrr}3,42 & 3,55 & 3,29 & 3,47 & \\ 3,73 & 4,76 & 4,66 & 4,51 & \\ 42,0 & 43,0 & 41,6 & 34,7 & \text { ESS-2007 } \\ 28,4 & 41,8 & 41,6 & 38,3 & \text { ESS-2007 } \\ 41,4 & 58,1 & 56,7 & 62,2 & \text { ESS-2007 } \\ 3,12 & 2,35 & 1,92 & 2,43 & \end{array}$

\section{D.3.1. Confianza institucional}

I8. Confianza en el sistema legal

(B5: suma categorías 6-10)

I9. Confianza en el Parlamento (B4: suma categorías 6-10)

I10. Satisfacción con el sistema democrático (B27: 6-10)

D.3.2. Participación en la vida política

I11. Interés por la política (B1: \% mucho + bastante)

I12. Le resulta fácil o dificil formarse una opinión sobre temas públicos (B3: ni difícil ni fácil + fácil + muy fácil)

$9,1 \quad 9,4 \quad 9,1 \quad 12,4 \quad$ ECVT-2004

$45,5 \quad 26,1 \quad 18,8 \quad 28,9 \quad$ ESS-2007 $63,0 \quad 50,6 \quad 41,4 \quad 53,5 \quad$ ESS-2007 
Cuadro 1. Estructura del índice sintético de capital social (continuación)

\begin{tabular}{llllll}
\hline Indicadores & Europa España And. Cat. Fuentes
\end{tabular}

I13. Se han puesto en contacto con una autoridad o funcionario estatal, autonómico o local (B13: \% sí) $\quad 13,0 \quad 12,0 \quad 11,7 \quad 11,0 \quad$ ESS-2007

I14. Ha colaborado con un partido político o una plataforma de acción ciudadana (B14: \% sí)

$3,5 \quad 5,1 \quad 4,9 \quad 3,7 \quad$ ESS-2007

D.4. Sociabilidad informal

$6,17 \quad 6,85 \quad 6,95 \quad 6,64$

- I15. Proporción de individuos que conviven con, al menos, otra persona (100 - \% individuos que viven solos)

- I16. Tasa de ocupación población mayor de 15 años

$\begin{array}{rrrrr}87,0 & 94,3 & 95,3 & 94,2 & \text { ECV-2004 } \\ 52,8 & 52,7 & 47,6 & 57,4 & \text { LFS }\end{array}$

- I17. \% se reúnen con amigos, familiares o compañeros de trabajo dos o más días por semana (p: C2: suma cat. 06-07)

\begin{tabular}{ccccc}
45,4 & 58,6 & 65,7 & 47,6 & ESS-2007 \\
\hline 2,99 & 2,57 & 1,48 & 3,34 &
\end{tabular}

Actitudes procívicas

— «Lograr que la gente pueda participar más en cómo se hacen las cosas en su lugar de trabajo o en su comunidad» ( v240: \% opción)

— «Dar a la gente mayor participación en las decisiones importantes del gobierno» (v242: \% opción)

$27,0 \quad 30,3 \quad 18,1 \quad 39,4 \quad$ ECVT-2004

Índice sintético de capital social $32,9 \quad 21,1 \quad 11,4 \quad 27,4 \quad$ ECVT-2004

Subíndice de capital social vinculante Subíndice de capital social aglutinante

$\begin{array}{llll}3,96 & 4,08 & 3,90 & 3,98 \\ 1,95 & 1,77 & 1,59 & 1,75 \\ 2,01 & 2,31 & 2,31 & 2,23\end{array}$

Fuentes: ESS, ECVT, FEDS, FIODS, ECV, EU-SILC y LFS.

tal (dimensiones) como el ISCS, se han calculado como suma promedio. Los resultados se han expresado en tanto por diez de forma tal que todos los subíndices toman valores entre 0 y 10 . La interpretación es sencilla y directa. Cero significa falta absoluta de cooperación, confianza social o participación cívica o, lo que es lo mismo, ausencia de sociedad. La puntuación diez reflejaría una intensa cooperación ciudadana en los asuntos de interés general o, lo que es lo mismo, la existencia de una auténtica sociedad civil. El ISCS se compone del índice de capital social vinculante (D1. Confianza social + D2. Participación en la vida social comunitaria + D3.2. Participación en la vida política) y del índice de capital social aglutinante (D3.1. Confianza institucional + D4. 
Cuadro 2. Índice y subíndices de capital social

\begin{tabular}{|c|c|c|c|c|c|c|}
\hline Indicadores & Europa & $\begin{array}{l}\text { Europa } \\
\text { occidental }\end{array}$ & $\begin{array}{l}\text { Europa } \\
\text { del Este }\end{array}$ & España & And. & Cat. \\
\hline $\begin{array}{l}\text { D.1. Confianza social } \\
((\mathrm{I} 1+\mathrm{I} 2+\mathrm{I} 3) / 3) * 0,1\end{array}$ & 4,07 & 4,40 & 3,46 & 3,81 & 3,57 & 3,76 \\
\hline $\begin{array}{l}\text { D.2. Participación en la vida } \\
\text { social comunitaria } \\
((\mathrm{I} 4+\mathrm{I} 5+\mathrm{I} 6+\mathrm{I} 7) / 4) * 0,1\end{array}$ & 2,20 & 2,44 & 1,76 & 2,11 & 1,81 & 2,05 \\
\hline $\begin{array}{l}\text { D.3. Participación en la vida } \\
\text { política (D.3.1. + D.3.2.)/2 } \\
\text { D.3.1. Confianza institucional }\end{array}$ & 3,42 & 3,63 & 3,04 & 3,55 & 3,29 & 3,47 \\
\hline $\begin{array}{l}((\mathrm{I} 8+\mathrm{I} \mathbf{9}+\mathrm{I} 10) / 3) * 0,1 \\
\text { D.3.2. Participación politica }\end{array}$ & 3,73 & 4,10 & 3,05 & 4,76 & 4,66 & 4,51 \\
\hline $\begin{array}{l}((\mathrm{I} 11+\mathrm{I} 12+\mathrm{I} 13+\mathrm{I} 14) / 4) * 0,1 \\
\text { D. } 4 . \text { Sociabilidad informal }\end{array}$ & 13,12 & 3,16 & 3,05 & 2,35 & 1,92 & 2,43 \\
\hline $\begin{array}{l}\qquad((\mathrm{I} 15+\mathrm{I} 16+\mathrm{I} 17) / 3) * 0,1 \\
\text { Actitudes procívicas }\end{array}$ & 6,17 & 6,30 & 5,93 & 6,85 & 6,95 & 6,64 \\
\hline $\begin{array}{l}x^{*} 0,1 \\
\text { Índice sintético de capitals social }\end{array}$ & 2,99 & 3,24 & 2,53 & 2,57 & 1,48 & 3,34 \\
\hline$((\mathrm{D} 1+\mathrm{D} 2+\mathrm{D} 3+\mathrm{D} 4) / 4)$ & 3,96 & 4,19 & 3,54 & 4,08 & 3,90 & 3,98 \\
\hline $\begin{array}{l}\text { Capital social vinculante (bridging) } \\
\text { Índice }\end{array}$ & 1,95 & 2,11 & 1,68 & 1,77 & 1,59 & 1,75 \\
\hline Proporción & 50,8 & 49,6 & 52,5 & 56,6 & 59,2 & 56,0 \\
\hline $\begin{array}{l}\text { Capital social aglutinante (bonding) } \\
\text { Índice }\end{array}$ & 2,01 & 2,08 & 1,86 & 2,31 & 2,31 & 2,23 \\
\hline Proporción & 50,8 & 49,6 & 52,5 & 56,6 & 59,2 & 56,0 \\
\hline
\end{tabular}

Fuentes: ESS, ECVT, FEDS, FIODS, ECV, EU-SILC y LFS. Elaboración propia. Nota: Europa: EU27, Europa occidental: EU15, Europa del Este: EU27-EU15.

Sociabilidad informal). También se ofrecen en este cuadro los datos que detallan las diferencias entre Europa occidental y Europa del Este.

El capital social, como cualquiera otra forma de capital, es acumulativo (Hanifan, 1916), de donde se deduce el interés por disponer del mayor volumen posible de capital social del tipo que sea. El capital social vinculante y el aglutinante no pueden maximizarse al mismo tiempo (Putnam, 2002) o, lo que es lo mismo, existe una correlación negativa entre ambos. En tercer lugar, una y otra formas de capital no son sustituibles (Inglehart, 2006), porque, puesto que tienen cualidades distintas, producirán efectos diferentes. En orden al desarrollo de la sociedad y en el escenario de la promoción de una auténtica sociedad civil, resulta más útil disponer de la mayor cantidad posible de capital social vinculante.

En consonancia con estos tres presupuestos, se compara, en primer lugar, el valor del índice sintético de capital social disponible en cada comunidad. 
El ISCS es equivalente al producto interior bruto social: lo que producen las relaciones sociales. En segundo lugar, analizamos las relaciones entre las dimensiones fuente del índice para valorar su contribución a la formación del capital social. Siguiendo con el símil económico, esto equivale a analizar la contribución al PIB (ISCS) de los diferentes sectores productivos (dimensiones del capital social). En tercer lugar, cuantificamos la contribución del capital social vinculante y aglutinante al stock global de capital. Valoramos lo que producen la sociabilidad formal y la sociabilidad informal, lo que, siguiendo con la metáfora económica, equivale a cuantificar lo que producen la economía formal y la economía informal. Por último, se ofrece una panorámica general y sintética de los distintos sistemas productivos de capital social. Este diagnóstico se basa en el análisis simultáneo del volumen (ISCS) y la composición del capital (índice de capital social vinculante e índice de capital social aglutinante).

\section{El capital social disponible (PIB social)}

El índice sintético de capital social (ISCS) representa el volumen global de recursos relacionales que posee una sociedad, el capital social disponible. El stock de capital social de que dispone la comunidad autónoma de Andalucía (ISCS Andalucía $=3,90)$ es prácticamente equivalente al de la comunidad autónoma de Cataluña (ISCS Cataluña $=3,98$ ). Ambas disponen de menos recursos sociales que el conjunto de España (ISCS España $=4,08$ ), que tampoco ocupa los primeros lugares en el ranking de los países de la Europa occidental (ISCS $\mathrm{UE} 15=4,19)$. Andalucía y Cataluña cuentan, sin embargo, con los mismos recursos que tienen, por término medio, el conjunto de los países europeos (ISCS UE27 $=3,96$ ).

No obstante, la diferencia entre el volumen global de capital social de Andalucía y de Cataluña no es significativa, por lo que se impone la prudencia a la hora de distinguir ambas regiones en términos de calidad de la sociedad. Según podemos apreciar en el cuadro 3, en el que se consignan las diferencias absolutas y relativas que existen en los valores del índice y de los subíndices para cada unidad territorial, la diferencia no sólo es pequeña, sino que es la mínima de las calculadas. El capital social disponible en Andalucía es, apenas, un $2 \%$ menor que el de Cataluña. Para interpretar las diferencias, se ha utilizado un criterio más restrictivo que el de su significatividad estadística: hemos definido como diferencias significantes aquellas diferencias relativas (DR) superiores al $10 \%$.

\section{Las dimensiones del capital social}

Entre las distintas dimensiones fuente del capital social, pueden observarse distintas correlaciones negativas, lo que confirma que unas y otras no pueden maximizarse al mismo tiempo. Y esto es así por la racionalidad del fenómeno, porque la participación requiere tiempo y esfuerzo. Los individuos tenderán 
Cuadro 3. Diferencias absolutas y diferencias relativas en las dimensiones del índice de capital social

\begin{tabular}{|c|c|c|c|c|c|c|c|}
\hline Dimensiones e índice & & $\begin{array}{l}\text { Andalucía/ } \\
\text { Cataluña }\end{array}$ & $\begin{array}{l}\text { Europa/ } \\
\text { Cataluña }\end{array}$ & $\begin{array}{l}\text { España/ } \\
\text { Andalucía }\end{array}$ & $\begin{array}{l}\text { UE15/ } \\
\text { España }\end{array}$ & $\begin{array}{l}\text { UE27/ } \\
\text { UE15 }\end{array}$ & $\begin{array}{l}\text { UE Este/ } \\
\text { España }\end{array}$ \\
\hline \multirow[t]{2}{*}{ D.1. Confianza social } & DA & $-0,19$ & 0,05 & 0,24 & 0,59 & $-0,33$ & $-0,35$ \\
\hline & DR & 5,3 & 1,3 & 6,3 & 13,4 & 8,0 & 10,10 \\
\hline \multirow{2}{*}{$\begin{array}{l}\text { D.2. Participación en la vida } \\
\text { social comunitaria }\end{array}$} & DA & $-0,24$ & 0,06 & 0,30 & 0,33 & $-0,24$ & $-0,35$ \\
\hline & DR & 13,2 & 2,8 & 14,2 & 13,5 & 10,9 & 19,9 \\
\hline \multirow{2}{*}{$\begin{array}{l}\text { D.3. Participación en la vida } \\
\text { política }\end{array}$} & DA & $-0,18$ & 0,08 & 0,26 & 0,08 & $-0,21$ & $-0,51$ \\
\hline & DR & 5,5 & 2,2 & 7,3 & 2,2 & 6,1 & 16,8 \\
\hline \multicolumn{2}{|c|}{ D.3.1. Confianza institucional DA } & 0,15 & 0,25 & 0,10 & $-0,66$ & $-0,37$ & $-1,71$ \\
\hline & $\mathrm{DR}$ & 3,2 & 5,2 & 2,1 & 16,1 & 9,9 & 56,1 \\
\hline \multirow{2}{*}{ D.3.2. Participación política } & DA & $-0,51$ & $-0,08$ & 0,43 & 0,81 & $-0,04$ & 0,70 \\
\hline & DR & 26,5 & 3,4 & 18,3 & 25,6 & 1,3 & 22,9 \\
\hline \multirow[t]{2}{*}{ D.4. Sociabilidad informal } & DA & 0,31 & 0,21 & $-0,10$ & $-0,55$ & $-0,13$ & $-0,92$ \\
\hline & DR & 4,5 & 3,1 & 1,4 & 8,7 & 2,1 & 15,5 \\
\hline \multirow[t]{2}{*}{ Actitudes procívicas } & DA & $-1,86$ & $-0,77$ & 1,09 & 0,67 & $-0,25$ & $-0,04$ \\
\hline & DR & 125,7 & 30,0 & 42,4 & 20,7 & 8,4 & 1,6 \\
\hline \multirow{2}{*}{$\begin{array}{l}\text { ISCS } \\
\text { (Índice sintético de capital social) }\end{array}$} & DA & $-0,80$ & 0,10 & 0,18 & 0,11 & $-0,23$ & $-0,54$ \\
\hline & $\mathrm{DR}$ & 2,0 & 2,4 & 4,4 & 2,6 & 5,8 & 15,2 \\
\hline \multirow[t]{2}{*}{ Índice de capital social vinculante } & DA & $-0,16$ & 0,02 & 0,18 & 0,34 & $-0,16$ & $-0,09$ \\
\hline & DR & 10,1 & 1,1 & 10,2 & 16,1 & 8,2 & 5,3 \\
\hline \multirow{2}{*}{ Índice de capital social aglutinante } & DA & 0,08 & 0,08 & 0 & $-0,23$ & $-0,07$ & $-0,45$ \\
\hline & $\mathrm{DR}$ & 3,5 & 3,5 & & 11,1 & 3,5 & 24,2 \\
\hline
\end{tabular}

Fuentes: ESS, ECVT, FEDS, FIODS, ECV, EU-SILC y LFS. Elaboración propia.

Nota: I: Índice, DA: Diferencia absoluta entre los índices, DR: Diferencia relativa entre los índices (\%). Europa: EU27, Europa occidental: EU15, Europa del Este: EU27-EU15.

a administrar estos recursos escasos en aras a alcanzar el mayor bienestar individual o colectivo posible. Esta razón determina la contribución de cada dimensión fuente del capital social al stock de capital disponible en una sociedad.

Al comparar los subíndices de cada dimensión (ver cuadro 2), se comprueba esta relación global negativa entre distintas dimensiones. Así, cuando la sociabilidad informal se hace máxima, desciende la participación en la vida política. Así, por ejemplo, la sociedad andaluza obtiene la máxima puntuación en sociabilidad informal (D4 $=6,95)$ y mínima en implicación política (D3 = 3,29). Así, en la Europa económicamente más desarrollada, la Europa de los quince, la menor importancia de las relaciones primarias como recurso de capital $(\mathrm{D} 4=6,30)$ se acompaña de una mayor participación política $(\mathrm{D} 3=3,63)$.

Esta misma relación se reproduce entre los dos componentes de la dimensión 3 (participación en la vida política): entre la confianza en las instituciones políticas del estado (D3.1) y la participación política directa (D3.2). En Europa, 
la menor confianza en las instituciones democráticas del estado se combina con una mayor disposición a implicarse directamente en los asuntos públicos. De otro modo sucede en Andalucía, en Cataluña y en la sociedad española en su conjunto, la mayor confianza en que las instituciones políticas del Estado actuarán de manera justa y no lesiva para los intereses de los ciudadanos, desincentiva la participación política. La confianza institucional desincentiva la participación porque tiende a generar un espíritu aquiescente con lo establecido. Además, la confianza institucional opera en sentido inverso a la confianza generalizada. Si la confianza interpersonal genérica (D1) es la condición sine qua non que invita a las personas a participar, la confianza en que las instituciones se conducirán de manera recta y respetarán lo establecido limita la participación.

Estas mismas relaciones surgen de la comparación entre ambas comunidades autónomas. En Cataluña, donde la participación política directa es mayor (D3.2 $=2,43)$, la sociabilidad informal desciende (D4 = 6,64). En Andalucía, donde la participación política es menor $(\mathrm{D} 3.2=1,92)$, la sociabilidad informal es mayor $(\mathrm{D} 4=6,95)$.

Tal y como puede apreciarse en el cuadro 3, en el que se detallan las diferencias significativas (marcadas en negrita), ambas comunidades se diferencian, en primer lugar, porque el subíndice de participación política directa calculado para Cataluña es un 26,5\% superior al de Andalucía y, en segundo lugar, porque la participación en organizaciones comunitarias es también superior en un $13,2 \%$.

La diferencia más ostensible es, sin lugar a dudas, la distinta valoración que unos y otros hacen de la participación. El subíndice que puede interpretarse como indicador de tendencia del capital social calculado para la comunidad autónoma catalana $(\mathrm{D} 5=3,34)$ es muy alto, e incluso superior al de la Europa occidental $(\mathrm{D} 5=3,24)$. Si los catalanes conceden mayor importancia a la participación social que los ciudadanos de los países de la Europa más desarrolla$\mathrm{da}$, pero confían mucho menos en la gente (D1 EU15 = 4,40 y D1 Cataluña $=3,76$ ), parece estar dibujándose un modelo de producción de capital social de corte individualista. Un sistema individualista en el que la implicación ciudadana en la producción del bien común no estaría intrínsecamente motivada, sino promovida por el temor a dejar las cuestiones colectivas en manos de representantes o ciudadanos poco responsables.

En Andalucía se valora muy poco la participación ciudadana $(\mathrm{D}=1,48)$. Esta escasa valoración se acompaña de una baja participación que se concreta en desinterés, desafección e inhibición de las decisiones de carácter político. Se combina, además, con una elevada confianza en las instituciones políticas del Estado. Según datos de la Encuesta Mundial de Valores aplicada en Andalucía en el año 1996, Del Pino y Bericat concluyen que la cultura andaluza concede mayor legitimidad al Estado y menor legitimidad a la sociedad civil. Puesto que las diferencias son lo suficientemente importantes, se puede hablar de una cultura más confiada en la estructuración política y social del Estado, que en la estructuración que provenga de la sociedad civil (Del Pino y Bericat, 1998: 289-290). 
El que se conceda prioridad a las instituciones de orden suele explicarse habitualmente por el mayor tradicionalismo y la menor presencia de los valores postmaterialistas en la cultura andaluza. La racionalidad que rige las relaciones sociales que producen capital, añade un elemento que facilita su interpretación. Si lo que la sociedad considera como metas prioritarias son preferentemente metas materialistas relacionadas con la estabilidad, y si los ciudadanos están convencidos de que las instituciones políticas del Estado garantizan esa cierta estabilidad social, no existirán motivos para implicarse directamente gastando tiempo y esfuerzo.

Los andaluces se inhiben de participar directamente en los asuntos políticos, porque confían en las instituciones democráticas depositarias de la soberanía popular, las instituciones que garantizan la participación indirecta. En este caso, no se aplica la estrategia del gorrón, que consiste en aprovecharse de los logros y las metas conseguidos por la participación directa de otros ciudadanos. Lo que reina es la conformidad y la satisfacción de que, sin coste añadido alguno para los ciudadanos, lo que logran las instituciones democráticas representativas es suficiente o, en todo caso, es más de lo que se podría conseguir con la participación directa. El modelo de producción del capital social de Andalucía logra la máxima eficiencia a base de no incurrir en ningún coste añadido y a condición de estar conforme con todo aquello de lo que nos provee el sistema democrático.

\section{Capital social vinculante y capital social aglutinante}

Nuestra hipótesis socioeconómica relaciona el predominio del capital social vinculante con el desarrollo y la innovación de la sociedad, y el capital social aglutinante con la subsistencia y la estabilidad del sistema social. Las dos formas del capital social pueden ser complementarias, pero no son intercambiables, porque cumplen funciones diferentes. Pero, supuesto que ambos tipos de capital no pueden maximizarse al mismo tiempo (Inglehart y Welzel, 2006) y que uno y otro no son sustituibles (Putnam, 2002), lo que resulta más rentable en términos de calidad de la sociedad es que, en la composición del capital global, predominen los lazos vinculantes, que ayudan a tender puentes, sobre los aglutinantes. Distinguimos, en este apartado, lo que produce la sociabilidad formal y la informal; lo que produce la participación política directa y la mediada.

En el cuadro 2, figura la contribución del capital social vinculante y aglutinante al stock global de capital. Aún cuando, tanto en Andalucía como en Cataluña, el peso del capital cívico es menor que el del capital primario, esta característica aparece mucho más acentuada en el caso andaluz. Tal y como observamos en el cuadro 3 , en el que aparecen las diferencias relativas, en Andalucía se produce un $10,1 \%$ menos de capital cívico que en Cataluña. Y, porque lo que sucede en Cataluña se parece mucho a lo que sucede en España, Andalucía aparece equidistante de ambas. Este predominio del capital social que obliga, sobre el que une y vincula, es una característica del mode- 
lo español $(\mathrm{CSV}=43,4 \%)$. En Europa, ambos componentes contribuyen casi por igual al stock de capital (CSV $=49,2 \%)$, incluso supera el $50 \%$ en el conjunto de países de la Europa occidental.

\section{Los modelos productivos de capital social}

Del análisis combinado del índice de capital social y de los índices específicos, se identifican tres sistemas productivos de capital social: el europeo, el de los países de la Europa del Este y el español. El modelo europeo se caracteriza por un elevado volumen de capital (ISCS $=3,96$ ) y un cierto equilibrio entre ambos componentes (vinculante $50 \%$ y aglutinante $50 \%$ ). Este es el modelo vigente en el conjunto de los 27 países de la Unión Europea y en los países de la Europa occidental. El rasgo típico del segundo modelo, el de los países de la Europa oriental, es que disponen de menos recursos sociales (ISCS: 3,54), con lo cual se observa un cierto equilibrio entre el capital social vinculante y aglutinante $(47,4 \%$ y $52,5 \%)$. El modelo español se parece al Europeo porque el capital social disponible es equivalente (ISCS $=4,08$ ), pero su singularidad se manifiesta en el mayor peso del capital aglutinante $(43,4 \%$ y $56,6 \%)$. Andalucía y Cataluña responden a los rasgos característicos del modelo español, aunque algunas ligeras diferencias nos llevan a pensar que se trate de dos variantes.

El modelo europeo se encuentra entre los más eficaces en la producción de capital social cívico. El modelo de la Europa del Este es el menos eficaz y heterodoxo. El español es un modelo eficaz de complementación entre lo que producen las tradicionales redes familiares y las organizaciones cívicas.

Entre los modelos analizados, el europeo es el más ortodoxo y equilibrado. Según la tesis de Inglehart, el capital social estaría cambiando de forma, el capital social vinculante se estaría haciendo cada vez más presente en detrimento del capital social aglutinante. Y es precisamente el modelo europeo aquel en el que el capital social cívico alcanza un mayor peso relativo. Podemos decir que es este el modelo más desarrollado. Atendiendo a las dimensiones del capital social, es también el más equilibrado. Todas las dimensiones varían en el sentido esperado: obtiene las mayores puntuaciones, tanto en confianza social como en participación comunitaria y política, y alcanza puntuaciones intermedias en la dimensión de sociabilidad informal.

Cuadro 4. Modelos producivos de capital social (stock y estructura productiva)

\begin{tabular}{|c|c|c|c|c|c|c|}
\hline & \multicolumn{2}{|c|}{ Modelo europeo } & \multicolumn{3}{|c|}{ Modelo español } & \multirow{2}{*}{$\frac{\text { Modelo Europa del Este }}{\text { UE27-UE15 }}$} \\
\hline & Europa & Europa occidental & España & Andalucía & Cataluña & \\
\hline & & & & & & 3,54 \\
\hline CS vinculante & $49,2 \%$ & $50,3 \%$ & $43,4 \%$ & $40,8 \%$ & $44,0 \%$ & $47,4 \%$ \\
\hline
\end{tabular}

Fuentes: ESS, ECVT, FEDS, FIODS, ECV, EU-SILC y LFS. Elaboración propia. 
Esta estructura productiva se parece más a la red que puede constituir una auténtica sociedad civil, porque las dimensiones fuente que producen el capital social cívico constituyen una trama equilibrada y porque la dimensión fuente del capital social que produce bienes privados, la sociabilidad informal, no tiene la suficiente autonomía para interferir sobre la primera. En un clima de confianza social, se da una elevada tasa de participación. Pero, además, en la dimensión que señala la participación en la vida política, se da un auténtico equilibrio entre una relativa confianza en las instituciones, necesaria para el funcionamiento del sistema democrático, y una activa participación política que garantiza la existencia de una opinión pública crítica. El equilibrio entre ambas subdimensiones es un elemento central del sistema, porque la confianza absoluta en las instituciones conduce a la apatía y desincentiva a la acción política.

Existe una verdadera trama que facilita la participación de ciudadanos intrínsecamente motivados, pero no existe una potente red paralela de sociabilidad primaria que pueda interferir, persiguiendo intereses privados, en la búsqueda del interés colectivo. La característica fundamental es que existe una sólida estructura productiva del capital social cívico y que no existe una potente estructura productiva paralela, como, por ejemplo, las redes de sociabilidad informal.

El modelo productivo de la Europa del Este es el menos eficaz, porque es el que menos capital social produce (ISCS $=3,54$ ). El capital vinculante y el aglutinante aportan el $50 \%$ al stock de capital o, lo que es lo mismo, uno y otro producen igual de poco. El equilibrio entre ambas formas de capital contrasta con el desequilibrio entre los componentes del capital. En comparación con los otros dos sistemas, el de la Europa del Este es el más heterodoxo, en la medida en que las dimensiones fuente de capital no correlacionan en el sentido esperado.

La mayor diferencia se establece en la dimensión "participación en la vida política». Su implicación política se define por una extrema desconfianza hacia las instituciones del Estado (UE27-15 = 3,05, UE15 = 4,10 y España = 4,76) y una relativamente alta participación política directa (UE27-15 $=3,05$, UE15 $=3,16$ y España $=2,35)$. Pero una elevada implicación política combinada con una desconfianza extrema, no implica necesariamente una actitud procívica. La participación puede limitarse a contestar y a protegerse de las arbitrariedades del sistema político, por lo que la actitud no será, desde ningún punto de vista, cooperativa.

El modelo español se basa en la complementariedad entre las fuentes de sociabilidad primaria y secundaria para la producción del capital social. Se trata de un sistema en el que la escasa producción del capital cívico se ve completada con lo que producen las redes primarias hasta alcanzar un nivel de capital tan elevado como el europeo. Puesto que una forma de capital social no puede sustituir a otra, la complementariedad es posible si, y solo si, ambas tienden a producir un mismo resultado: el bienestar colectivo. No podría darse semejante modelo en el caso de que las formas de solidaridad primaria, fuertemente arraigadas y culturalmente institucionalizadas, propiciaran la hostilidad hacia el extraño. Así, por ejemplo, las redes de sociabilidad informal del sur de Italia difícilmente podrían integrarse en un modelo de complementariedad. 
En un sistema en el que prima el familismo amoral, las redes familiares y de amistad contribuyen a generar círculos de solidaridad cerrados y aislados de la sociedad. Por el contrario, en un sistema familista como el español, las redes primarias, lejos de contribuir a la exclusión, integran a los individuos en la sociedad. Conseguir un empleo a través de familiares, de amigos o de conocidos; conseguir llegar a fin de mes con una pensión de jubilación o un subsidio de desempleo regresando al hogar familiar, o mantener a los hijos treintañeros mientras consiguen integrarse en el segmento del mercado laboral de los «buenos empleos», son ejemplos de cómo las redes primarias favorecen la integración de los individuos en la sociedad, con lo cual contribuyen a promover el mayor bienestar colectivo.

Para producir y acumular capital social, es necesario hacer un esfuerzo activo, esto es, de manera análoga a como sostiene la teoría del capital económico, requiere que se haga una inversión (Kadushin, 2004). La teoría de la acción racional postula que los actores pueden estar motivados, bien hacia la salvaguarda de los recursos existentes o bien hacia la consecución de recursos alternativos. La inversión que se hace para mantener los recursos que ya existen es menor que la que se necesita para lograr nuevos recursos. Cuando las redes primarias producen recursos a fin de satisfacer muchas de las necesidades de los individuos, la motivación por buscar recursos alternativos desciende. Así, la motivación por participar en redes de acción colectiva puede mantenerse en niveles bajos, porque esta participación requiere más tiempo y más esfuerzo que el que resulta necesario para mantener activas las redes de solidaridad primarias. Disponer de una sólida red de reciprocidad familiar, como en el modelo español, no sólo no promueve el espíritu cívico que conduce a la participación en organizaciones, sino que también la desincentiva.

\section{Los modelos productivos de capital social en Andalucía y Cataluña}

Los rasgos principales de los modelos catalán y andaluz coinciden con los del modelo español, pero se observan, sin embargo, ciertas e importantes singularidades. El modelo catalán se distingue, en primer lugar, porque produce más capital social vinculante que el andaluz (Cataluña: 44,0\% y Andalucía: 40,8\%) $y$, en consecuencia, menos capital social aglutinante. Se produce más capital cívico porque se participa más en organizaciones en beneficio de la comunidad (DR: $13,2 \%$ ) y porque se está más directamente implicado en los asuntos políticos (DR: 26,5\%). Es en Cataluña donde se dan las mejores condiciones para la formación de una opinión pública crítica, allí donde existe una mayor proporción de ciudadanos interesados por los asuntos políticos, y donde más fácil les resulta a muchos forjarse una opinión política.

Al contrario también de lo que sucede en Andalucía, es en la comunidad autónoma catalana donde menos se invierte en el mantenimiento de las redes primarias de reciprocidad. Los catalanes son los que se reúnen con menor frecuencia con amigos, familiares y compañeros de trabajo, el 47,6\% declara hacerlo dos o más días por semana, mientras que en España esta proporción 
asciende al 58,6\% y se eleva en Andalucía hasta el 65,7\%. Y no sólo se invierte menos, sino que es donde el coste de mantener las redes primarias se percibe como más oneroso. Así, los catalanes perciben que participan mucho más que la media en este tipo de actividades sociales, mientras los andaluces piensan que participan mucho menos que sus conciudadanos. La relación de este indicador con el coste de la actividad se explica por un mecanismo de carácter psicosocial por el que, una vez que se ha decidido no hacer algo, se tiende a elevar subjetivamente el coste de dicha actividad. Así, por ejemplo, aquellos individuos que nunca han tenido hijos piensan que el coste es más elevado, mientras que aquellos que tienen hijos creen que el coste es menor. Quizá piensan que gastan mucho más tiempo en actividades sociales, porque sus prioridades son otras.

El que se invierta menos en mantener las redes primarias puede deberse también a que les reporta menos beneficios, por ejemplo, a la hora de conseguir un empleo. Si el coste de mantenimiento es elevado y los beneficios de la participación son menores, estos ciudadanos serán más proclives a buscar otros recursos de capital social.

El modelo andaluz destaca porque acentúa el rasgo más característico del modelo español: la complementariedad. La brecha entre el capital vinculante y el aglutinante se hace mucho más profunda en esta comunidad $(59,2 \%$ y $40,8 \%$ ). Tienen un mayor peso los recursos sociales producidos en los grupos primarios y, de manera especial, en la familia. Y es que si la cultura española es familista, la andaluza lo es todavía más. En un artículo, en el que tratamos de responder a la pregunta a propósito de ¿qué es lo que quieren decir los españoles cuando afirman que la familia es muy importante?, concluíamos que la familia es muy importante porque satisface necesidades básicas, frecuentes y perentorias de los individuos (Camarero, 2002).

Los andaluces invierten mucho tiempo y esfuerzo en mantener estos vínculos primarios: son los que más asisten a ferias y lugares de encuentro (el 63,7\% de los andaluces asistió a alguna feria en el último año frente al 41,0\% de los catalanes), los que con mayor frecuencia se reúnen con amigos y familiares y los que, sin embargo, consideran que se dedican menos que la mayoría de la gente a todas estas actividades sociales. Y si estas actividades producen recursos (véase, por ejemplo, que casi la mitad de los trabajadores señaló haber conseguido el empleo a través de familiares, amigos y/o conocidos), existirá un fuerte interés por mantener la red. La elevada confianza en los miembros de los grupos de solidaridad primaria tiende a generar desconfianza en los otros, pero la confianza es necesaria para participar en todo tipo de asociaciones promotoras del bien común. En Andalucía, la confianza interpersonal necesaria para la creación del capital social cívico es baja.

En segundo lugar, es de destacar el desequilibrio que se produce entre la elevada satisfacción con las instituciones del Estado y la baja implicación en la vida política. La elevada conformidad con el sistema establecido junto con la escasa voluntad por participar directamente en las decisiones públicas indica que el proceso de comunicación entre las instituciones representativas del 
Estado y los ciudadanos no es lo suficientemente fluido. No parecen darse las condiciones para que los ciudadanos expresen públicamente sus opiniones: porque hay un escaso o nulo interés por la política (sólo dos de cada diez andaluces dijo estar muy interesado o bastante interesado en política) y porque tienen dificultades para formarse una opinión (el 60\% señaló que le resulta difícil o muy difícil formarse una opinión). Este es el indicador más evidente de un déficit de calidad democrática de la sociedad.

Tanto el modelo catalán como el andaluz pueden considerarse como dos variantes del modelo español de complementariedad. Un modelo en el que lo que ya producen las redes primarias, no tiene por qué ser producido a través de redes formales. Algunos indicadores, y especialmente los que expresan el valor que los catalanes conceden a la participación social y política, señalan que éste puede estar tomando un nuevo rumbo cívico. El sistema andaluz se caracteriza por exacerbar el rasgo más definitorio del modelo español, la prevalencia del capital social aglutinante. Esta variante extrema del modelo de complementariedad se presenta como un sistema, casi un engranaje podríamos decir, perfectamente ajustado y estable.

\section{Referencias bibliográficas}

AGUiAR, Fernando (1998). «Bienes públicos». En: GINER, Salvador; LAMO DE EsPinOSA, Emilio y TORRES, Cristóbal (eds.). Diccionario de sociología. Madrid: Alianza.

BERGER-SCHMITT, Regina (2002). «Considering social cohesion in quality of life assessments: Concept and measurement». Social Indicators Research, 58 (1-3).

BERICAT, Eduardo y CAMARERO, Mercedes (2008). Sistema de indicadores para la monitorización del cambio social en Andalucía. Sevilla: Instituto de Estadística de Andalucía (inédito).

BURT, Ronald (1997). «The contingent value of social capital». Administrative Science Quarterly, 42 (2), 339-365.

CAMARERO, Mercedes (2002). «La familia española contemporánea. Funcionalidad y relevancia». En: VVAA. La sociedad: Teoría e investigación empirica. Madrid: Centro de Investigaciones Sociológicas.

ColEmAN, James (1988). «Social capital in the creation of human capital». The American Journal of Sociology, 94, 95-120.

- (1994). Foundations of social theory. Cambridge: Harvard University Press.

CRUDELI, L. (2006). «Social capital and economic opportunities». The Journal of SocioEconomics, 35, 913-927.

DAHRENDORF, Ralf (1996). La cuadratura del círculo: Bienestar económico, cohesión social y libertad política. México: Fondo de Cultura Económica.

Donat, Pierpaolo y PrandinI, Riccardo (2007). «The family in the light of a new relational theory of primary, secondary and generalized social capital». International Review of Sociology, 17 (2), 209-223.

FuKUYAMA, Francis (2000). «Social capital». En: HARRISON, L.E. y Hungtington, S.P. (eds.). Culture matters. How values shape human progress. Nueva York: Basic Books.

FuKUYAMA, Francis (2001). «Social capital, civil society and development». Third World Quarterly, 22 (1), 7-20. 
GitTell, Ross y Vidal, A. (1998). Community organizing: Building social capital as a development strategy. Thousand Oaks: Sage.

GOBERNADO, Rafael (coord.) (1996). Análisis comparado de las estructuras sociales de Andalucía y Cataluña. Málaga: Universidad de Málaga.

GrANOVETTER, Mark (1985). «Economic action and social structure: The problem of embeddedness». American Journal of Sociology, 91 (3), 481-510.

Hanifan, Lyda Judson (1916). "The Rural School Community Center». Annals of the American Academy of Political and Social Science, 67, 130-138.

Helliwell, J. y PuTNAM, Robert (2000). «Economic growth and social capital in Italy». En: Social capital. Washington, DC: World Bank, 253-268.

Herreros, Francisco (2002). "¿Son las relaciones sociales una fuente de recursos?: Una definición del capital social». Papers, 67, 129-148.

- (2004). The problem of forming social capital: Why trust? Nueva York: Palgrave.

INGLEHART, Ronald (2006). Modernización, cambio cultural y democracia: la secuencia del desarrollo humano. Madrid: Centro de Investigaciones Sociológicas.

IngleHART, Ronald y Welzel, Ch. (2000). «Culture and Democracy». En: HARRISON, L.E. y Hungtington, S.P. (eds.). Culture matters: How values shape human progres. Nueva York: Basic Books.

JAIME, Antonio (2005). «La compleja relación entre el capital social y confianza política desde el sur de Europa. Evidencia empírica en Andalucía». En: ANDRÉU, Jaime (coord.). Desde la esquina de Europa: Análisis comparado del capital social en Andalucía, España y Europa. Sevilla: Centro de Estudios Andaluces, 57-87.

Kadushin, Ch. (2004). "Too much investment in social capital?». Social Networks, 26, 75-90.

Luhmann, Niklas (1996). Confianza. Barcelona: Universidad IberoamericanaAnthropos.

Montero, José Ramón; ZMerLi, S. y Newton, K. (2008). "Confianza social, confianza política y satisfacción con la democracia». Revista Española de Investigaciones Sociológicas, 122, 11-54.

Noll, Heinz Herbert (2002). "Towards a European system of social indicators: Theoretical framework and system architecture». Social Indicators Research, 58 (13), 47-63.

Onyx, Jenny y Bullen, Paul. (1998). " Measuring Social Capital in Five Communities in NSW: An Analysis». Centre for Australian Community Organizations and Management. Working Papers Series no41.

PAHL, Ray (2003). Sobre la amistad. Madrid: Siglo XXI.

PATULNY, R.V. (2007). «Exploring the social capital grid: bonding, bridging, qualitative, quantitative». International Journal of Sociology and Social Policy, 27 (1-2), 32-51.

PAXTON, Pamela (1999). "Is social capital declining in the United States?: A multiple indicator assessment». American Journal of Sociology, 105 (1), 88-127. - (2002). "Social capital and democracy: an interdependent relationship». American Sociological Review, 67, 254-277.

PÉREZ DÍAZ, Víctor (2003). «De la guerra civil a la sociedad civil: el capital social en España entre los años treinta y los años noventa del siglo XX». En: PUTNAM, R.D. (ed.). El declive del capital social: Un estudio internacional sobre las sociedades y el sentido comunitario. Barcelona: Galaxia Gutemberg, 427-489.

PINO, Juan del y BERICAT, Eduardo (1998). Valores sociales en la cultura andaluza: Encuesta Mundial de Valores. Andalucía 1996. Madrid: Centro de Investigaciones Sociológicas. 
Portes, Alejandro y Sensenbrener, J. (1993). «Embeddedness and inmigration: Notes on the social determinants of economic action». American Journal of Sociology, 98 (6), 1320-1350.

Putnam, Robert (1993). Making democracy work: Civic traditions in modern Italy. Princeton: Princeton University Press.

- (2000). Bowling alone: The collapse and revival of American Community. Nueva York: A Touchstone Book.

- (2002). Solo en la bolera: Colapso y resurgimiento de la comunidad norteamericana. Barcelona: Galaxia Gutemberg.

- (ed.) (2003). El declive del capital social: Un estudio internacional sobre las sociedades y el sentido comunitario. Barcelona: Galaxia Gutemberg-Círculo de Lectores.

Requena, Félix (2004). "El capital social en la Encuesta de Calidad de Vida en el Trabajo». Papers, Revista de Sociología, 73, 11-26.

Rose, Richard (2006). First European Quality of Life Survey: Participation in civil society. Dublín: European Foundation for the Improvement of Living and Working Conditions.

Rosenberg, M. (1956). "Misantrhopy and political ideology». American Sociological Review, 6, 690-695.

SCHEFfler, R.M.; Brown, T.T. y Rice, J.K. (2007). "The role of social capital in reducing non-specific psychological distress: The importance of controlling for omitted variable bias». Social Science \& Medicine, 65 (4), 842-854.

SzTOMPKA, Piotr (2006). "Review essay. New perspectives on trust». American Journal of Sociology, 112 (3), 905-919.

TorCal, Mariano (2008). «El origen y la evolución del apoyo a la democracia en España: La construcción del apoyo incondicional en las nuevas democracias». Revista Española de Ciencia Política, 18, 29-65.

Whiteley, P.F. (2000). «Economic growth and social capital». Political Studies, 48, 443-466.

WoOlCCOCK, M. (1998). «Social capital and economic development: Toward a theoretical synthesis and policy framework». Theory and Society, 27, 151-208.

\section{Fuentes estadísticas}

European SCIENCE Foundation. European Social Survey. [http://www.europeansocialsurvey.org]

Eurostat. EU Survey on Income and Living Conditions, EU-SILC. [http://epp.eurostat.ec.europa.eu/portal/page/portal/eurostat/home/]

EUROSTAT. Labor Force Survey, LFS. [http://epp.eurostat.ec.europa.eu/portal/page/portal/eurostat/home/]

Federación Española de Donantes de Sangre. Contabilidad de las Asociaciones provinciales de Donantes de Sangre. [http://www.donantesdesangre.net]

Federación Internacional de Organizaciones de Donantes de Sangre. Contabilidad de las Asociaciones Nacionales de Donantes de Sangre. [http://www.fiods.org]

INE. Encuesta de Condiciones de Vida, ECV. [http://www.ine.es]

INE. Encuesta de Población Activa, EPA. [http://www.ine.es]

Ministerio de Trabajo e Inmigración. Encuesta de Calidad de Vida en el Trabajo, ECVT. [http://www.mtas.es/] 